\title{
Reconstrucciones volumétricas de hueso temporal mediante Cone Beam (CBCT)
}

\author{
Volumetric reconstruction of temporal bone by Cone Beam (CBCT)
}

\author{
Ilson Sepúlveda A'.
}

\begin{abstract}
RESUMEN
La Tomografía Computarizada tipo Cone Beam (CBCT) ha demostrado ser una herramienta radiológica muy útil en el campo de la otorrinolaringología. Es una técnica tridimensional con bajos niveles de radiación, lo que implica un menor número de artefactos e igual o mayor resolución en comparación con la Tomografía Computarizada Multicorte (MSCT). Hoy en día es una excelente opción para la exploración radiológica del oído.
\end{abstract}

Palabras clave: Cone Beam, CBCT, hueso temporal, reconstrucción, volumétrica.

\begin{abstract}
Cone Beam Computed Tomography (CBCT) has proven to be a useful radiological tool in the field of otolaryngology. It is a three dimensional technique with low levels of radiation, which implies fewer artifacts and equal or greater resolution compared with Multislice Computed Tomography (MSCT). Today is an excellent choice for the radiological examination of the ear.
\end{abstract}

Key words: Cone Beam CBCT, temporal bone, reconstruction, volumetric.

La Tomografía Computarizada tipo ConeBeam (CBCT), es una técnica radiológica relativamente nueva $^{1-3}$. Fue desarrollada en la década de los años 70 y utilizada en imagen vascular hasta los primeros años de la década de los años $80^{4,12}$. CBCT se ha utilizado principalmente en odontología, en particular para la evaluación de posibles sitios receptores implantes dentales, de patologías de los maxilares y en traumatología del complejo maxilofacial2,5,7.
La técnica se caracteriza por la adquisición rápida de imágenes de alta resolución en tres dimensiones, baja exposición a radiación y menos artefactos en comparación con la tomografía computarizada multicorte (MSCT). Las unidades de CBCT usan intensificadores de imagen 0 sensores flat-panel. El voxel es isotrópico y la resolución varía entre 0,07 a 0,25 mm. Estos valores superan a la mayoría de las unidades de CT multicorte, en términos de resolución espacial,

1 Servicio de Otorrinolaringología, Cirugía de Cabeza y Cuello, Hospital Clínico Regional de Concepción. 
análisis de imágenes en tiempo real y visualización tridimensional ${ }^{1,5,6}$.

Específicamente, respecto a la comparación dosimétrica, en miliGrays (mGy), basado en el Índice de Dosis de Tomografía Computarizada (CTDI) entre CBCT y MSCT, Dahmani-Causse y cols., reportaron en adquisiciones unilaterales de hueso temporal, valores promedio de 175,83 mGy para MSCT; y de $8,17 \mathrm{mGy}$ para $\mathrm{CBCT}^{13}$.

El examen se puede realizar con el paciente de pie o sentado, haciendo que el procedimiento sea cómodo, ideal para niños y adultos claustrofóbicos. El problema específico de formación de imágenes en CBCT es el movimiento del paciente, que disminuye la calidad de toda la exploración ${ }^{2,7}$.

El uso de CBCT ha ido ganando aceptación en aplicaciones para otorrinolaringología (ORL) y actualmente está siendo utilizado para la adquisición de imágenes del hueso temporal, fracturas de huesos propios nasales y evaluación de las cavidades perinasales ${ }^{1,2,5}$. Estudios recientes también han explorado la utilidad de CBCT en la evaluación de la pérdida progresiva de la audición. Dalchow y cols evaluaron mediante CBCT a 25 pacientes con pérdida de audición conductiva, confirmada mediante audiometría preoperatoria y concluyó que la CBCT podría ser muy precisa, tanto en la predicción de la integridad como en la detección de erosiones de la cadena osicular ${ }^{10}$ (Figura 1).

Otra de las ventajas en el valor diagnóstico de la CBCT fueron reportadas en estudios que analizaron la visualización y posición de prótesis en oído medio, las que pueden determinarse con gran precisión. Esto aporta información valiosa para el diagnóstico, planificación preoperatoria y prevención de complicaciones intraoperatorias ${ }^{2,3,6}$ (Figura 2).

En oído interno, la CBCT es eficaz en el diagnóstico de la mayoría de las malformaciones, displasias, lesiones traumáticas, erosión, adelgazamiento o dehiscencia del laberinto óseo y control de la posición de implantes cocleares ${ }^{2,5,8,9}$ (Figuras 3 y 4 ).

Gupta y cols demostraron que la visualización estructural con CBCT fue significativamente mayor en comparación con las de MDCT. Estructuras particularmente bien visualizadas incluyen la cadena osicular, el laberinto óseo del oído interno, anatomía coclear y trayecto del nervio facial. Sugieren que la falta de contraste de los tejidos blandos en sus evaluaciones no interfirió con precisión diagnóstica debido al gran número de estructuras de alto contraste ubicadas en el hueso temporal y el efecto positivo de la mayor resolución espacial en algunas estructuras de bajo contraste, tales como el nervio facial11 (Figura 5).

\section{CONCLUSIÓN}

Comparado con el MSCT en hueso temporal, la CBCT es una excelente alternativa, con bajos niveles de radiación, libre de artefactos, cortes superfinos, alta resolución y más efectivo, si la indicación es precisa.

\section{BIBLIOGRAFÍA}

1. Saeed Fakhran y cols. Comparison of Simulated Cone Beam Computed Tomography to Conventional Helical Computed Tomography for Imaging of Rhinosinusitis. Laryngoscope 2014 Jan 21.

2. Afshin Teymoortash y cols. Temporal bone imaging using digital volume tomographyand computed tomography: a comparative cadaveric radiological study. Surg Radiol Anat 2011; 33: 123-8.

3. Robert MLYNSKI y cols. Presentation of floating mass transducer and Vibroplasty Couplers on CT and Cone Beam CT. Eur Arch Otorhinolaryngol 2014; 271: 665-72.

4. CYNThia C. Leung Y cols. Accuracy and reliability of cone-beam computed tomography for measuring alveolar bone height and detecting bony dehiscence and fenestrations. American Journal of Orthodontics and Dentofacial Orthopedics 2010; 137: 109-18.

5. DBS LADEIRA Y COLS. Prevalence and characteristics of pneumatization of the temporalbone evaluated by cone beam computed tomography. Int J Oral Maxillofac Surg 2013; 42: 771-5.

6. HAMDI CAKLI Y COLS. Use of cone beam computed tomography in otolaryngologic treatments. Eur Arch Otorhinolaryngol 2012; 269: 711-20

7. Hanzelka T y cols. Movement of the patient and the cone beam computed tomgraphy scanner: objectives and possible Solutions. Oral and 


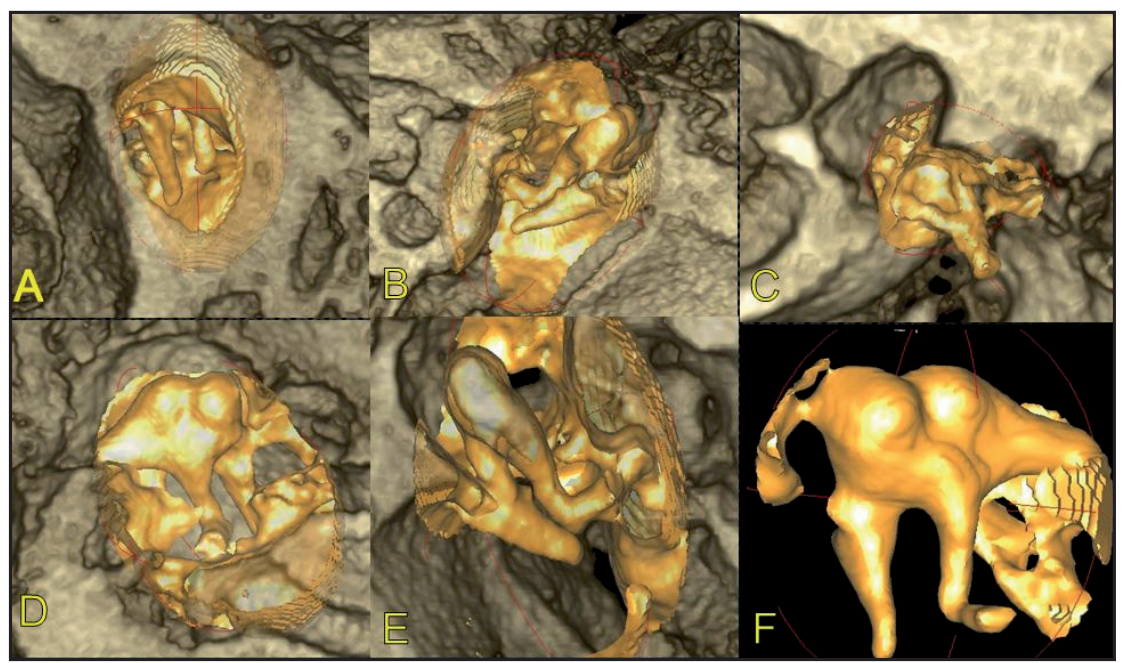

Figura 1. Reconstrucción volumétrica 3D de la cadena osicular. A: vista lateral; B: vista anterior; C: estribo; D: vista medial; E: vista posterior; F: articulación incudo-maleolar.

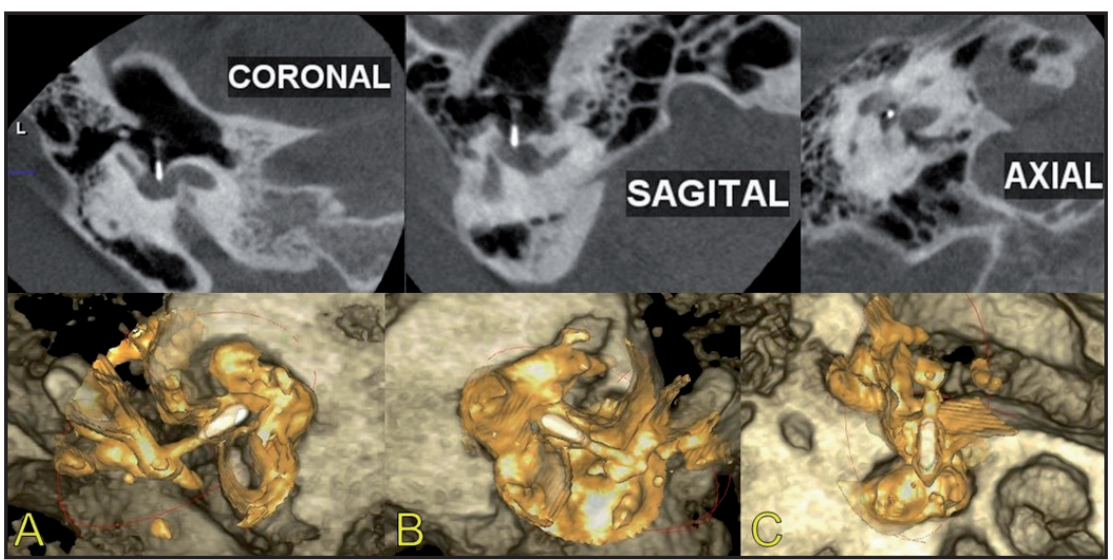

Figura 2. Control poscirugía del estribo. Reconstrucción volumétrica 3D. A: vista anterior; B: vista posterior; C: vista superior.

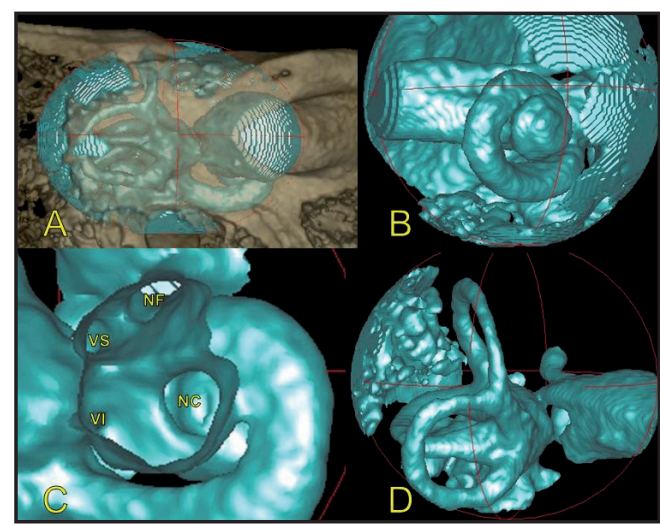

Figura 3. Reconstrucción volumétrica 3D con algoritmo de partes blandas. A: oído interno. B: cóclea. C: CAI (VS: vestibular superior; NF: nervio facial; VI: vestibular inferior; NC: nervio coclear). D: canales semicirculares.

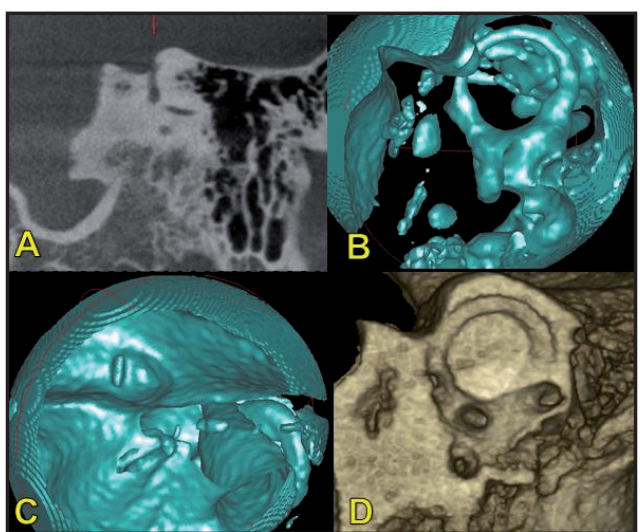

Figura 4. Canal semicircular superior dehiscente. A: vista coronal; B: reconstrucción 3D con algoritmo cavidad aérea; C: vista endocraneal; D: reconstrucción 3D con algoritmo óseo. 


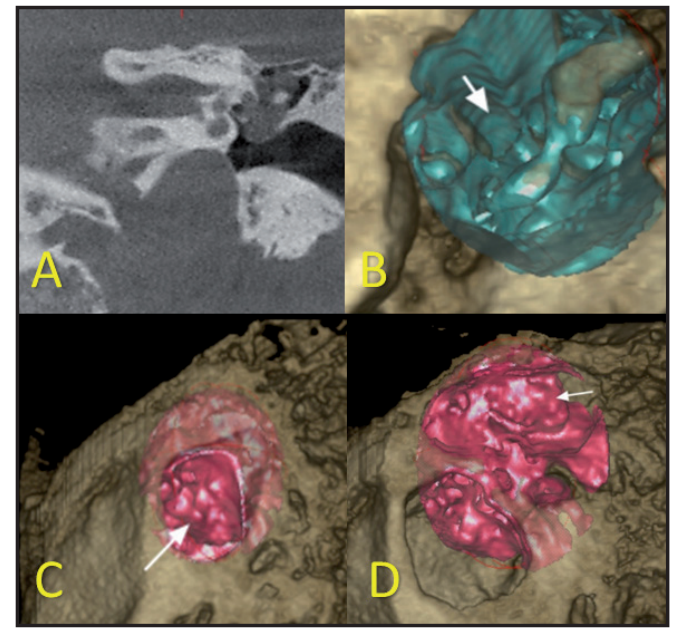

Figura 5. Colesteatoma ático-antromastoideo. A: vista coronal; B: ausencia pared lateral, segmento timpánico VII par; C: reconstrucción 3D algoritmo partes blandas, otoscopía virtual; D: ocupación del antro mastoideo.

Maxillofacial Radiology 2013; 116(6): 769-73.

8. CV DaLCHOW Y COLS. Radiographic examination of patients with dehiscence of semicircular canals with digital volumen tomography. Eur Arch Otorhinolaryngol 2013; 270: 511-9.

9. XIN LIANG Y COLS. A comparative evaluation of Cone Beam Computed Tomography (CBCT) and Multi-Slice CT (MSCT). Part II: On 3D model accuracy. European Journal of Radiology 2010; 75: 270-4.

10. Dalchow CV y cols. Value of digital volume tomography in patients with conductive hearing loss. Eur Arch Otorhinolaryngol 2006; 263: 92-9.
11. GUPTA $R$ y cols. Experimental flat panel highspatial resolution volume CT of the temporal bone. Am J Neuroradiol 2004; 25: 1417-24.

12. ANIKET By cols. Classification and volumetric analysis of temporal bone pneumatization using cone beam computed tomography. Oral Surg Oral Med Oral Pathol Oral Radiol 2014; 117: 376-84.

13. M Dahmani-Causse y cols. Morphologic examination of the temporal bone by cone beam computed tomography: Comparison with multislice helical computed tomography. European Annals of Otorhinolaryngology, Head and Neck diseases 2011; 128: 230-5.

Dirección: Ilson Alexis Sepúlveda A.

E mail: isesepulvedaguilar@gmail.com 